\title{
Momentum and temperature dependence of renormalization effects in the high-temperature superconductor $\mathrm{YBa}_{2} \mathrm{Cu}_{3} \mathrm{O}_{7-\delta}$
}

\author{
V. B. Zabolotnyy, ${ }^{1}$ S. V. Borisenko, ${ }^{1}$ A. A. Kordyuk, ${ }^{1,2}$ J. Geck, ${ }^{1}$ D. S. Inosov, ${ }^{1}$ A. Koitzsch, ${ }^{1}$ J. Fink, ${ }^{1}$ M. Knupfer, ${ }^{1}$ \\ B. Büchner, ${ }^{1}$ S.-L. Drechsler, ${ }^{1}$ H. Berger, ${ }^{3}$ A. Erb, ${ }^{4}$ M. Lambacher, ${ }^{4}$ L. Patthey,${ }^{5}$ V. Hinkov, ${ }^{6}$ and B. Keimer ${ }^{6}$ \\ ${ }_{1}^{1}$ Institute for Solid State Research, IFW-Dresden, P. O. Box 270116, D-01171 Dresden, Germany \\ ${ }^{2}$ Institute of Metal Physics of National Academy of Sciences of Ukraine, 03142 Kyiv, Ukraine \\ ${ }^{3}$ Institute of Physics of Complex Matter, EPFL, CH-1015 Lausanne, Switzerland \\ ${ }^{4}$ Walther-Meißner-Institut, Bayerische Akademie der Wissenschaften, Walther-Meißner Strasse 8, 85748 Garching, Germany \\ ${ }^{5}$ Swiss Light Source, Paul Scherrer Institut, CH-5234 Villigen, Switzerland \\ ${ }^{6}$ Max-Planck Institut für Festkörperforschung, D-70569 Stuttgart, Germany
}

(Received 1 February 2007; revised manuscript received 9 July 2007; published 15 August 2007)

\begin{abstract}
We present a detailed angle-resolved photoemission (ARPES) study of the low-energy electronic structure of $\mathrm{YBa}_{2} \mathrm{Cu}_{3} \mathrm{O}_{7-\delta}$ and show that the ARPES spectrum generally contains contribution from two components, of which the prevailing one is hugely overdoped, while the other retains superconductivity and represents the bulk properties. The doping level of the overdoped surface component turns out to be weakly dependent on the oxygen stoichiometry, always remaining close to $x \sim 0.3$. By suppressing the overdoped component, we clearly show that the other one develops a superconducting gap consistent with a $d$-wave symmetry. As in $\mathrm{Bi}_{2} \mathrm{Sr}_{2} \mathrm{CaCu}_{2} \mathrm{O}_{8+\delta}$, the superconducting component is marked by the unusual renormalization which is strongly enhanced at antinodes and vanishes above $T_{C}$, supporting the universality of the conclusion regarding the magnetic origin of the unusual renormalization in high- $T_{C}$ superconductors.
\end{abstract}

DOI: 10.1103/PhysRevB.76.064519

PACS number(s): 74.25.Jb, 74.72.Hs, 79.60.-i

Angle-resolved photoelectron spectroscopy (ARPES) plays an important role in providing detailed information about many-body effects and the electronic structure of hightemperature superconductors, and together with other experimental methods and theoretical efforts facilitated a noticeable advance in our understanding of high-temperature superconductivity (HTSC). In particular, appreciable contributions to establish the overall picture in HTSC have recently been made by investigations of the charge dynamics in $\mathrm{Bi}_{2} \mathrm{Sr}_{2} \mathrm{CaCu}_{2} \mathrm{O}_{8+\delta}(\mathrm{Bi}-2212)$ (Refs. 1-3) and the spin dynamics in $\mathrm{YBa}_{2} \mathrm{Cu}_{3} \mathrm{O}_{7-\delta}$ (Y-123), ${ }^{4-8}$ using ARPES and inelastic neutron scattering (INS), respectively. These advances, in turn, have stimulated a set of works in which the authors, combining the experimental data with theoretical efforts, endeavored to interrelate particular features clearly observed in the INS spectrum of Y-123 with those seen in the ARPES spectrum of Bi-2212. ${ }^{9,10}$ The next obvious step forward could be use of the experimental data sets for the same compound, preferably Y-123 in a view of the higher quality of INS data for this compound. ${ }^{4-7}$ Unfortunately, ARPES on Y-123 turned out to be a complicated technique producing rather perplexing results even for the basic electronic structure, not to mention the finer effects in the spectral function, like the superconducting gap or unusual renormalization, clearly detected in $\mathrm{Bi}-2212 .^{2,3,11-13}$

While in one of the earliest works ${ }^{16}$ it is claimed that both bonding and antibonding bands are clearly detected and that these bands are forming extending along $\Gamma-\mathrm{X}$ and $\Gamma-\mathrm{Y}$ direction saddle points with binding energies of about 19 and $150 \mathrm{meV}$, in Refs. 17 and 18 it is suggested that the feature at the $\mathrm{Y}$ point with binding energy around $19 \mathrm{meV}$ belongs to a surface state that arises from chains, crippled after cleavage and that the antibonding and bonding bands have significantly higher binding energies of about 120 and $660 \mathrm{meV}$, respectively. More recent work, ${ }^{19}$ performed with significantly improved instrumental resolution, touches upon another acute problem of ARPES on Y-123, which is observation of a sizable superconducting gap. The solution suggested there was to reattribute the feature that is observed in the vicinity of the $\mathrm{X}$ and $\mathrm{Y}$ points and has the lowest binding energy to a surface state of uncertain origin, thus explaining the absence of a sizable leading edge gap (LEG) in ARPES. However, the remaining features were discussed in terms of a peak-dip-hump structure, which is difficult to reconcile with the expectation of the bilayer split bands as predicted by the local density approximation LDA. ${ }^{20}$

Only very recent studies ${ }^{21-23}$ arrived at a certain level of consistency concerning the experimentally observed electronic structure of Y-123. It is shown that for the nearly stoichiometric $\mathrm{YBa}_{2} \mathrm{Cu}_{3} \mathrm{O}_{7-\delta}$ the photoemission spectrum consists of two components. One of the components represents the bulk bonding and antibonding bands that are retaining superconductivity and, presumably, have the nominal bulk doping level. The other component arises from the bilayer nearest to the cleavage, which turns out to be strongly overdoped and thus displays no signatures of superconductivity in the ARPES spectrum. Although the solution suggested by Nakayama et al. ${ }^{22,23}$ turns out to be an important finding for a better understanding of ARPES on Y-123, the mere statement that the overdoped component is localized at the very topmost bilayer, while the superconducting one comes from the bulk states, needs to be justified, as a similar picture might also arise from lateral inhomogeneities at the cleaved surface. Regardless of the superconducting and overdoped components' localization, the more important question that makes this compound still interesting and waits for a definitive answer is whether the strong renormalization effects, clearly observed in Bi-2212, ${ }^{11,13}$ are also inherent to Y-123. 

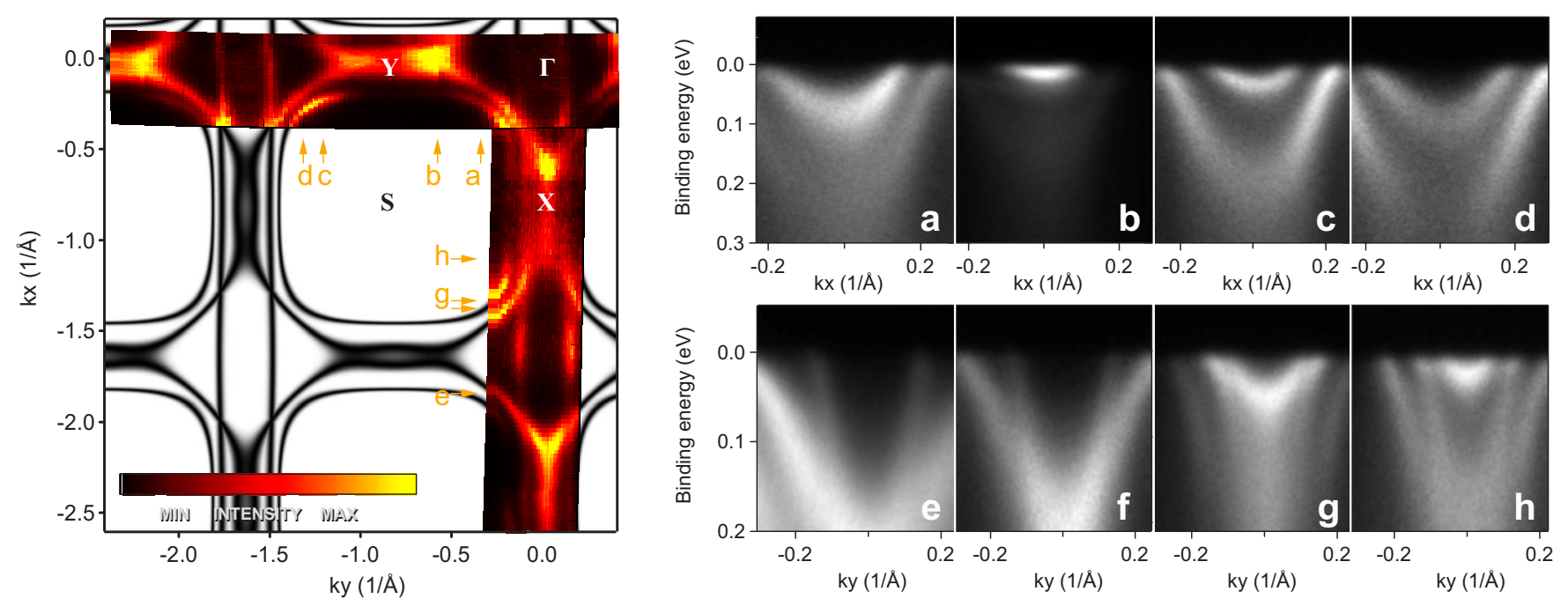

FIG. 1. (Color online). Experimental band structure of untwinned $\mathrm{YBa}_{2} \mathrm{Cu}_{3} \mathrm{O}_{6.85}, T_{C}=90 \mathrm{~K}$. The left panel contains two experimental FS maps measured along the $\Gamma$-X and $\Gamma$-Y directions and the tight-binding fit to them. (a)-(d) Energy-momentum intensity maps at various $k_{x}$ values indicated in the FS map, $h \nu=50 \mathrm{eV}$. The projection of $k_{y}$ for these spectra was held constant; its value is denoted by arrows with corresponding lettering on the FS map. (e)-(h) Similar measurements, taken after rotating the sample by $90^{\circ}$ around the $\Gamma$ point, $h \nu$ $=55 \mathrm{eV}$. The image (e) is the sum of spectra measured with circularly polarized light of left and right helicities. The other spectra were measured with linearly polarized light.

In this study, having done extensive measurements over the whole Brillouin zone, we confirm the issue of the surface overdoping. Afterward, we address the question of renormalization effects in Y-123, which might be masked by the intense overdoped component. To tackle the problem of the overdoped component we consider two approaches. (i) If for nearly stoichiometric samples with hole doping level close to the optimal the surface bilayer turns out to be strongly overdoped, one could expect a lower doping level for the dominating surface component in the case of nominally underdoped samples. Thus spectra with optimal doping can be recorded from the surface component, while the weak photoemission intensity from the deeper $\mathrm{CuO}_{2}$ bilayers can be simply neglected. (ii) It was shown that overdoping of the near surface bilayer is intimately related to the cleavage interface of Y-123, which for the stoichiometric compound is known to be preferably located between the $\mathrm{CuO}$ and $\mathrm{BaO}$ layers. ${ }^{24-26}$ However, there is also evidence for other cleavage interfaces ${ }^{27,28}$ though the probability of such an event is very small. With this in mind, one might expect to have a different cleavage interface stabilized for the samples with modified stoichiometry. In particular, here we investigate the effect of Ca substitution for $\mathrm{Y}$ atoms.

The data were collected at the SIS beamline at the Swiss Light Source using the SCIENTA SES 100 electron analyzer. Samples were mounted on a high-precision cryomanipulator and cleaved in situ in an ultrahigh-vacuum chamber with base pressure of $5 \times 10^{-11} \mathrm{mBar}$. All spectra, except for the Fermi surface (FS) maps, were measured with an energy resolution of $12 \mathrm{meV}$ and an angular resolution of $0.2^{\circ}$. For mapping purposes the energy resolution was set to $20 \mathrm{meV}$. The excitation energies and temperatures are stated within the corresponding spectra or in the figure captions. The Fermi level was calibrated using the cold Fermi edge of a fresh silver film evaporated on the sample at the end of the experiment. High-quality single crystals were synthesized by the solution-growth technique and annealed to the desired oxygen content. Here we present the data for the nearly optimally doped samples $\mathrm{YBa}_{2} \mathrm{Cu}_{3} \mathrm{O}_{6.85} \quad\left(T_{C} \approx 90 \mathrm{~K}\right)$ and $\mathrm{YBa}_{2} \mathrm{Cu}_{3} \mathrm{O}_{6.4} \quad\left(T_{C} \approx 35 \mathrm{~K}\right)$, which were untwinned by uniaxial mechanical stress at elevated temperatures. The critical temperatures for the twinned $\mathrm{YBa}_{2} \mathrm{Cu}_{3} \mathrm{O}_{6.9}$ and $\left(\mathrm{Y}_{1-x} \mathrm{Ca}_{x}\right) \mathrm{BaCu}_{3} \mathrm{O}_{7-\delta}, \quad x \approx 0.15$ were 90 and $77 \mathrm{~K}$ respectively.

We start the discussion with a brief description for the $\mathrm{YBa}_{2} \mathrm{Cu}_{3} \mathrm{O}_{6.85}$ band structure as seen in ARPES spectrum. Figure 1 shows the experimental Fermi surface map for near optimally doped untwinned $\mathrm{YBa}_{2} \mathrm{Cu}_{3} \mathrm{O}_{6.85}$. In contrast to many previous studies, we use higher excitation energies (50-60 eV vs $15-30 \mathrm{eV}$ ) that enhance the intensity of the Fermi level (FL) crossings of both bonding and antibonding bands and allow examination of a larger area in $k$ space. The visual agreement with the band structure calculations ${ }^{20}$ is remarkable. There are two holelike sheets of the Fermi surface centered around the $S$ point. A set of one-dimensional features running along the $\Gamma-\mathrm{X}$ direction is a clear indication for the presence of states related to the chain substructure of Y-123. Even the form of the antibonding sheet of the FS develops W-like undulations close to the $\mathrm{Y}$ point. It is also remarkable that, unlike $\mathrm{Bi}-2212,{ }^{29}$ the bilayer splitting along the FS is more isotropic, so that even along the nodal $\Gamma$-S cut its value remains comparable to that along the antinodal Y-S cut. To further substantiate identification of the bands, several typical intensity plots are provided in the Figs. 1(a)-1(h). Each of these plots shows the photo intensity as a function of binding energy and momentum along selected directions in $k$ space with the bright features tracing band dispersion. Here, in analogy to another bilayer compound $\mathrm{Bi}-2212$, one can follow the behavior of the plane-derived bonding and the antibonding bands. The spectra in the Figs. 

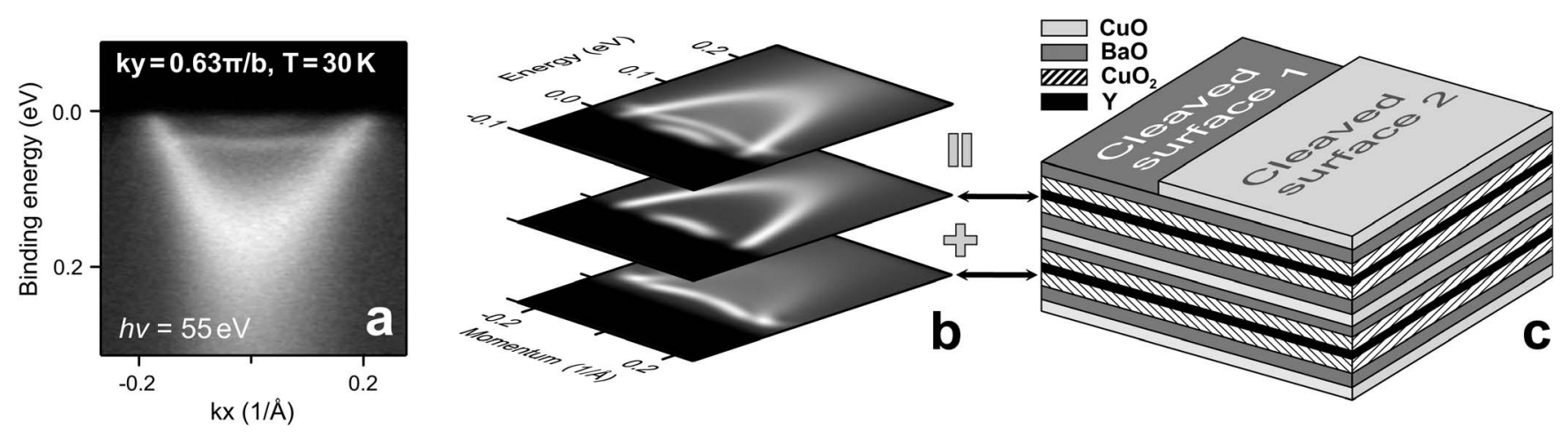

FIG. 2. Coexistence of superconducting and metallic components. (a) Experimental spectrum comprising the contribution from normal and superconducting states for $\mathrm{YBa}_{2} \mathrm{Cu}_{3} \mathrm{O}_{6.85}, k_{y}=0.63 \pi / b, T=30 \mathrm{~K}, h \nu=55 \mathrm{eV}$. (b) Model image, to explain the spectrum as a superposition of the signals from superconducting and overdoped (metallic) bilayers. The bottommost image is the spectrum of the superconducting phase, middle, the metallic, and the topmost image their sum. (c) Schematic structure of the cleaved crystal. The doping of the $\mathrm{CuO}_{2}$ bilayer nearest to the surface is modified due to disturbed chains ( $\mathrm{CuO}$ and $\mathrm{BaO}$ layers), while the next bilayer remains unimpaired and retains superconductivity.

1(e)-1(h) show the band dispersion along the $k_{y}$ axis, where one-dimensional chain bands are expected. Indeed, the corresponding paraboliclike band remains unchanged in all four cuts, giving rise to the pairs of lines parallel to the $k_{x}$ axis on the Fermi surface map. In Fig. 1(e) the chain band is enveloped by the antibonding band and part of the bonding band can be seen in the left bottom corner dispersing beyond the image frame. The images of Figs. 1(f) and 1(g) demonstrate how the $\mathrm{CuO}_{2}$-plane-derived bands cross the FL closer and closer to the $\Gamma-\mathrm{X}$ line when moving toward the $\mathrm{X}$ point, so that finally the chain band can be found between the bonding and antibonding bands. It looks as if the relative position of the chain band with respect to the plane bands is not much different from the one predicted by the LDA. Therefore we find a good qualitative agreement between our data and LDA calculations $^{20}$ for the electronic structure of Y-123, although the data do not support the existence of the predicted $\mathrm{Ba}-\mathrm{O}-$ derived pocket centered at the $\mathrm{S}$ point. Despite qualitative agreement between the observed FS and the LDA predictions, quantitative estimate of the hole doping based on the FS area, ${ }^{30}$ in accordance with the recent finding by $\mathrm{Na}-$ kayama et $a .^{22,23}$ reveals strong overdoping with $x \approx 0.31$. This provides a natural explanation for the absence of the signatures of superconductivity such as the superconducting gap and typical BCS-like band bending, or abrupt breaks in the band velocity in the antinodal region detected for Bi-2212. ${ }^{11}$

It also turns out that the Y-123 spectra are not only limited to the overdoped component. Changing the photon energy by only $5 \mathrm{eV}(h \nu=55 \mathrm{eV})$ transforms the photoemission intensity as found in Fig. 1(b) into the spectrum presented in Fig. 2(a). An extra component is easily detected in the vicinity of the $\mathrm{X}$ and $\mathrm{Y}$ points, where it looks like a nondispersing flat feature with a binding energy of about $40 \mathrm{meV}$. This feature strongly resembles the mode-renormalized and gapped spectrum of Bi-2212, ${ }^{13}$ when in the superconducting state the spectral weight is confined in a narrow energy range between the gap and the onset of scattering by a collective mode. ${ }^{14,15}$ This feature gives rise to an energy distribution curve (EDC) consisting of three peaks, which has previously been reported in Ref. 19 (see also Fig. 3). At that time the metallic component was interpreted differently: Its antibonding band was believed to be a "surface peak," while the term "hump" was used for the bonding band. This feature appears universally in the superconducting state of all Y-123 samples with various doping levels that we have studied. Our temperaturedependent measurements (see Fig. 3), in agreement with previous results, ${ }^{19,22,23}$ unambiguously show that the feature virtually disappears above $T_{C}$, indicating an intimate relationship with superconductivity. We attribute this feature mainly to the antibonding band of the nominally doped bulk component. In Fig. 2(b), using model spectra, we demonstrate how the superposition of superconducting and metallic spectra results in the rather complex structure observed in the experiment [Fig. 2(a)].

To understand the observed superposition of the two component in the spectra, the matter of the cleavage plane has to be addressed in more detail. Existing data ${ }^{24-26}$ indicate that the preferable cleavage for Y-123 is the plane between the $\mathrm{Cu}-\mathrm{O}$ and $\mathrm{Ba}-\mathrm{O}$ layers, which leads, in principle, to two types of surface termination [Fig. 2(c)]. Since for the Y-123 it is the amount of oxygen $\mathrm{O}(1)$ (Ref. 27) in the chains that drives the doping level of the neighboring $\mathrm{CuO}_{2}$ bilayers, such a cleavage would destroy the chains and certainly change the doping level of the adjacent bilayers. Therefore one plausible explanation could be that the two components arise from these two types of surface termination [see Fig. 2(c)]. However, we believe this scenario to be unlikely. If the size of the patches with fixed doping was larger than the beam size, this should result in variation of the intensity ratio between the two components when moving the beam over the sample, which is not supported by our observation. Assuming the size of the patches to be much smaller than the beam diameter $(\varnothing 100 \mu \mathrm{m})$ also does not help much, as this implies statistically equal total areas corresponding to the overdoped and nominally doped patches, and hence comparable intensity of the two component, which is again not supported by the experimental data. The fact that the over- 

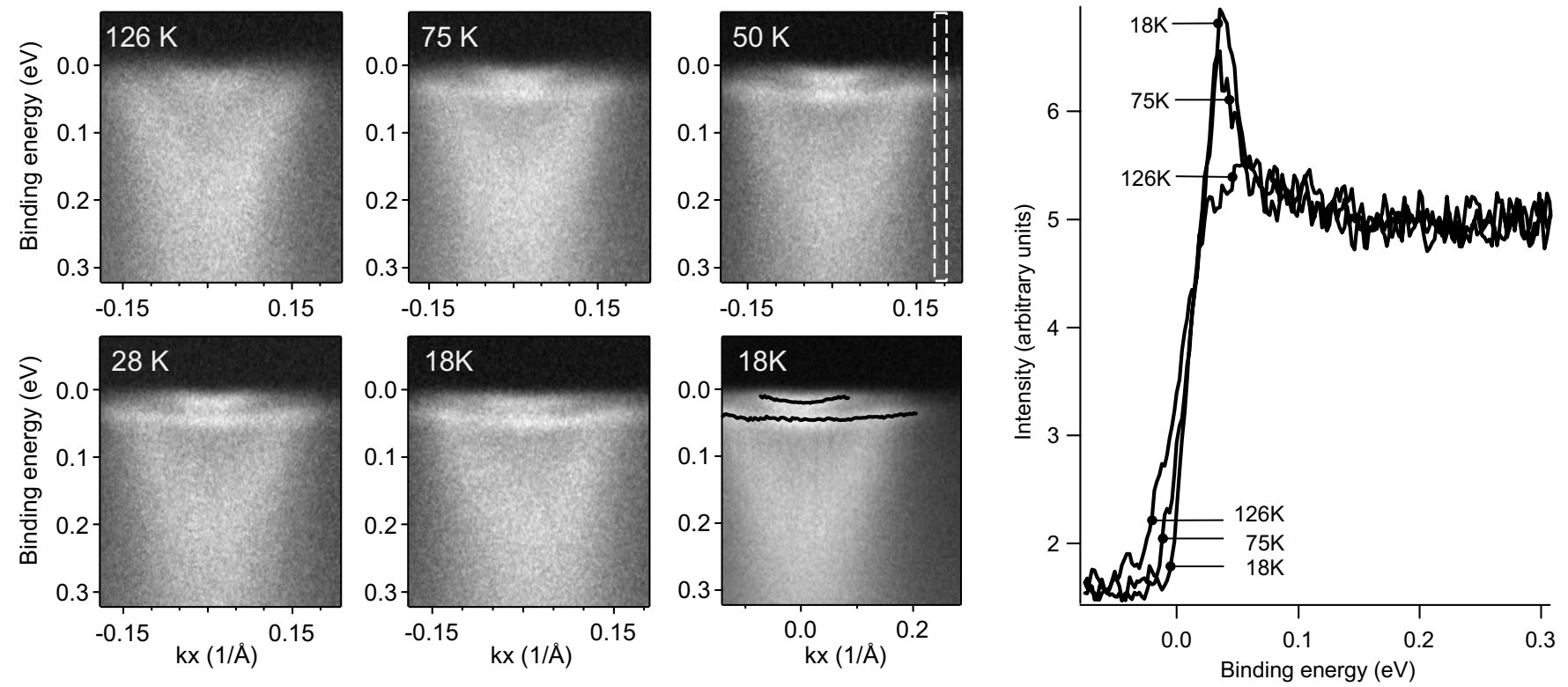

FIG. 3. Temperature dependence of the superconducting feature for $\mathrm{YBa}_{2} \mathrm{Cu}_{3} \mathrm{O}_{6.9}$. The right hand image shows evolution of the EDCs integrated in a small momentum window. The EDCs corresponding to 28 and $50 \mathrm{~K}$ have been omitted as they practically overlap with the $18 \mathrm{~K}$ one. The integration range is delimited by a dashed rectangle plotted on top of the $50 \mathrm{~K}$ spectrum. The black curves on top of the $18 \mathrm{~K}$ spectrum trace the dispersions of the overdoped antibonding band (the upper one) and the superconducting feature (the lower one).

doped component turns out to be always dominating rather suggests that the superconducting component comes from the deeper regions from under the cleavage plane. Taking into account the small electron escape depth, we are inclined to believe that the overdoped component is localized in the bilayer nearest to the surface, while the superconducting component arises mainly from the next bilayer, the doping level of which remains unchanged. Additional evidence to locate the overdoped component at the surface bilayer comes from our recent study of circular dichroism in Y-123. There we show that the states localized in the near surface region become sensitive to circularly polarized light, resulting in strong circular dichroism due to substantial contribution of the term $\operatorname{divA}$ in the photoemission matrix element. ${ }^{32}$

Now we proceed to the question of overcoming the hindering effect of the overdoped component. Expecting to get the overdoped surface component, whose doping level resides within the superconducting dome, we concentrated on samples with lower bulk doping level, $\mathrm{YBa}_{2} \mathrm{Cu}_{3} \mathrm{O}_{6.6}$ and $\mathrm{YBa}_{2} \mathrm{Cu}_{3} \mathrm{O}_{6.4}$. Figure 4 summarizes the results for the sample with the lowest doping, close to the antiferromagnet boundary on the phase diagram. As can be seen in Fig. 4, there is a notable effect on the size of the Fermi surface. However, accurate counting of the FS area results in a hole doping of
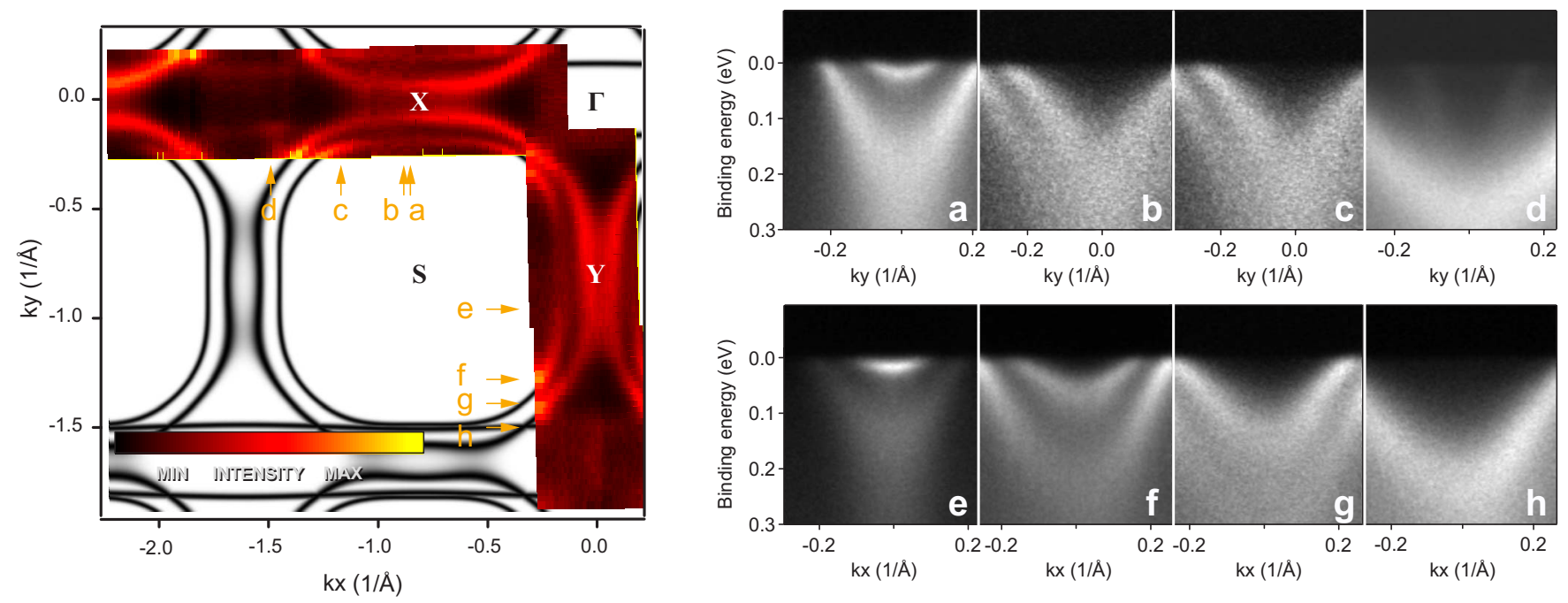

FIG. 4. (Color online) Experimental band structure of untwinned $\mathrm{YBa}_{2} \mathrm{Cu}_{3} \mathrm{O}_{6.4}, T_{C}=35 \mathrm{~K}$. The data layout is the same as in Fig. 1; all spectra were recorded at $30 \mathrm{~K}$. The FS map and the separate cuts (b)-(h) were measured with linear polarization and $h \nu=50 \mathrm{eV}$. For the image (a) linearly polarized light with $h \nu=55 \mathrm{eV}$ was used. 

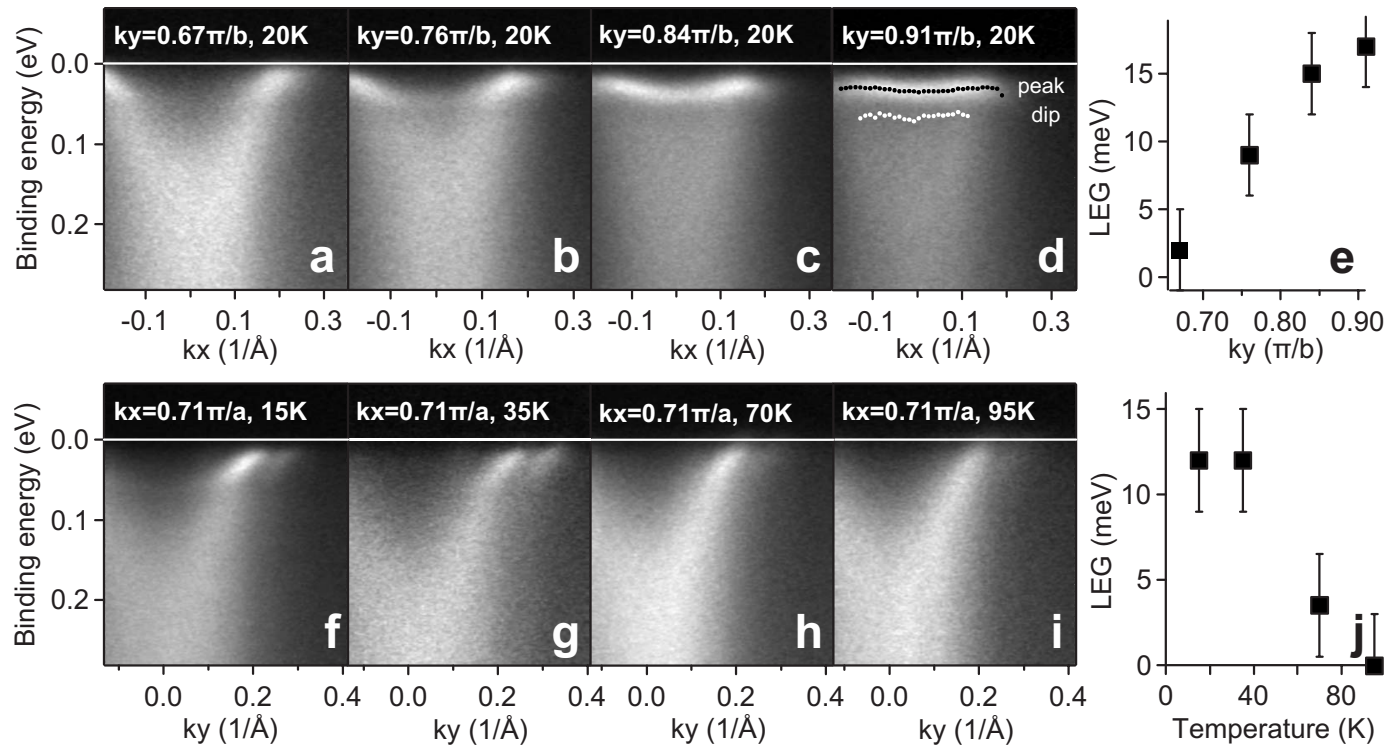

FIG. 5. Superconducting component. (a)-(d) Opening of a superconducting gap and enhancement of the band renormalization effects when approaching the antinodal point. Symbols in the image (d) denote the peak and the dip positions extracted from the EDC's comprising this data set. (e) Extracted value of the superconducting gap. (f)-(i) Evolution of the spectral weight and the leading edge gap (j) as a function of temperature. The spectra were measured from a freshly cleaved $\left(\mathrm{Y}_{1-x} \mathrm{Ca}_{x}\right) \mathrm{BaCu}_{3} \mathrm{O}_{7-\delta},\left(x=0.15 \pm 0.03, T_{C}=77 \mathrm{~K}\right)$ using linearly polarized light, $h \nu=50 \mathrm{eV}$.

about 0.27 , which is still too much for either clear detection of the superconducting gap or notable renormalization effects in the spectra of the surface component. From this we have to conclude that there is some internal mechanism that keeps the doping level of the surface layer at a saturation value of about 0.3 , regardless of the bulk hole doping level.

Thus we pass on to the second possibility, which is investigation of samples with modified stoichiometry. In particular, we have studied partially Ca-substituted Y-123 samples. In Fig. 5 we show the spectra for $\left(\mathrm{Ca}_{x} \mathrm{Y}_{1-x}\right) \mathrm{BaCu}_{3} \mathrm{O}_{7-\delta},(x$ $=0.15 \pm 0.03)$, where the superconducting component dominates. The samples were initially grown for the INS scattering experiments and the amount of substituted $\mathrm{Ca}$ was a compromise between the quality of the samples and the effect of $\mathrm{Ca}$ atoms on the doping level. In Figs. 5(a)-5(e) one can directly observe the momentum dependence of the leading edge gap value with its anisotropic character consistent with $d$-wave symmetry. The temperature evolution of the gap is given in Figs. 5(f)-5(j) and leaves no doubt as to the superconducting nature of these spectra. A very important observation here is the occurrence of renormalization anomalies in the band dispersion and their strong momentum and temperature dependence. It is notable that there are practically no renormalization effects due to the mode coupling for the overdoped component [compare to Figs. 1(a)-1(h); Fig. $5(\mathrm{~g})$ contains a visible admixture of the antibonding band of the superconducting component], which demonstrates a substantial doping dependence of the renormalization. It is these dependences that were used to argue that the reason for the unusual renormalization effect in Bi-2212 is the coupling to magnetic excitations. ${ }^{13,31}$ As can be seen in Figs. 5(c) and $5(d)$, the strong renormalization in the vicinity of the $(\pi, 0)$ point results in the suppression of the spectral weight at certain energy, so that single EDCs extracted from these images would develop a so-called peak-dip-hump line shape. According to the spin fluctuation theory ${ }^{14,15}$ one can ascribe this to coupling to the INS resonance, and its energy can be estimated as the peak-to-dip distance. For the data in Fig. 5(d) we fitted the peak and dip positions and estimated the resonance energy to be about $33 \pm 3 \mathrm{meV}$, which is in agreement with the data for $\left(\mathrm{Y}_{0.85} \mathrm{Ca}_{0.15}\right) \mathrm{Ba}_{2} \mathrm{Cu}_{3} \mathrm{O}_{7}\left(T_{C}=75 \mathrm{~K}\right)$ reported in Ref. 8. This proves the conclusions about the mode origin made earlier for Bi-2212 to be equally applicable for the Y-123 case, hence backing their generality.

To account for the prevalence of the superconducting component for the Ca-substituted sample, in addition to the matrix element effects, one needs to assume the appearance of another cleavage plane. The analysis of the crystal structure suggests that the additional cleavage is most likely to happen within the $\mathrm{Y}$ layer, since in this case the topmost $\mathrm{CuO}_{2}$ plane would be too strongly disrupted to give spectra similar to those in the bulk (including but not limited to the bilayer splitting), while the bilayer nearest to the surface, being practically undisturbed and sharing the charge from both adjacent chain layers, would result in a signal corresponding to the superconducting component. In view of the different ionic radii of $\mathrm{Ca}(1.18 \AA)$ and $\mathrm{Y}(0.89 \AA)$, the cleavage over the $\mathrm{Y}$ layer appears favorable also due to the slackening introduced by $15 \%$ Ca substitution; however, tunneling data may be necessary to irrevocably settle this particular issue concerning $\mathrm{Ca}$ substitution.

To conclude, we have shown that the main stumbling block on the way to understand spectra of Y-123 was the presence of two components - a superconducting and a metallic one. Our data are consistent with the model where these two components coexist in two neighboring bilayers at the surface. We also show that lowering of the surface 
component doping via a decrease of the bulk doping level does not seem to be viable. However, the overdoping effect can be completely removed via the use of Ca-substituted Y-123 samples. We show that the superconducting component in Y-123 possesses all the necessary attributes as in other HTSCs, namely, an anisotropic superconducting gap and band renormalization effects that depend on momentum and doping level and vanish at $T_{C}$; therefore we support the generality of the mentioned effects. Having understood the Y-123 spectra, the next promising step is to use ARPES data and INS results obtained for the same compound to make a quantitative check for consistency of theories aiming to explain high- $T_{C}$ superconductivity.

This project is part of the Forschergruppe FOR538 and was supported by the DFG under Grant No. KN393/4 and by BMBF under Grant No. 05KS40D218. This work was performed at Swiss Light Source, Paul Scherrer, Villigen, Switzerland. We thank R. Hübel for technical support and M. Rümmeli for careful reading of the manuscript.
${ }^{1}$ A. Damascellil, Z. Hussain, and Z.-X. Shen, Rev. Mod. Phys. 75, 473 (2003).

${ }^{2}$ P. V. Bogdanov, A. Lanzara, S. A. Kellar, X. J. Zhou, E. D. Lu, W. J. Zheng, G. Gu, J.-I. Shimoyama, K. Kishio, H. Ikeda, R. Yoshizaki, Z. Hussain, and Z. X. Shen, Phys. Rev. Lett. 85, 2581 (2000).

${ }^{3}$ A. Kaminski, M. Randeria, J. C. Campuzano, M. R. Norman, H. Fretwell, J. Mesot, T. Sato, T. Takahashi, and K. Kadowaki, Phys. Rev. Lett. 86, 1070 (2001).

${ }^{4}$ P. Bourges, Y. Sidis, H. F. Fong, L. P. Regnault, J. Bossy, A. Ivanov, and B. Keimer, Science 288, 1234 (2000).

${ }^{5}$ H. F. Fong, P. Bourges, Y. Sidis, L. P. Regnault, J. Bossy, A. Ivanov, D. L. Milius, I. A. Aksay, and B. Keimer, Phys. Rev. Lett. 82, 1939 (1999).

${ }^{6}$ H. A. Mook, Pengcheng Dai, S. M. Hayden, G. Aeppli, T. G. Perring, and F. Doğan, Nature (London) 395, 580 (1998).

${ }^{7}$ V. Hinkov, S. Pailhès, P. Bourges, Y. Sidis, A. Ivanov, A. Kulakov, C. T. Lin, D. P. Chen, C. Bernhard, and B. Keimer, Nature (London) 430, 650 (2004).

${ }^{8}$ S. Pailhès, C. Ulrich, B. Fauque, V. Hinkov, Y. Sidis, A. Ivanov, C. T. Lin, B. Keimer, and P. Bourges, Phys. Rev. Lett. 96, 257001 (2006).

${ }^{9}$ U. Chatterjee, D. K. Morr, M. R. Norman, M. Randeria, A. Kanigel, M. Shi, E. Rossi, A. Kaminski, H. M. Fretwell, S. Rosenkranz, K. Kadowaki, and J. C. Campuzano, Phys. Rev. B 75, 172504 (2007).

${ }^{10}$ D. S. Inosov, S. V. Borisenko, I. Eremin, A. A. Kordyuk, V. B. Zabolotnyy, J. Geck, A. Koitzsch, J. Fink, M. Knupfer, B. Büchner, H. Berger, and R. Follath, Phys. Rev. B 75, 172505 (2007).

${ }^{11}$ T. K. Kim, A. A. Kordyuk, S. V. Borisenko, A. Koitzsch, M. Knupfer, H. Berger, and J. Fink, Phys. Rev. Lett. 91, 167002 (2003).

${ }^{12}$ P. D. Johnson, T. Valla, A. V. Fedorov, Z. Yusof, B. O. Wells, Q. Li, A. R. Moodenbaugh, G. D. Gu, N. Koshizuka, C. Kendziora, Sha Jian, and D. G. Hinks, Phys. Rev. Lett. 87, 177007 (2001).

${ }^{13}$ S. V. Borisenko, A. A. Kordyuk, T. K. Kim, A. Koitzsch, M. Knupfer, M. S. Golden, J. Fink, M. Eschrig, H. Berger, and R. Follath, Phys. Rev. Lett. 90, 207001 (2003).

${ }^{14}$ Ar. Abanov and A. V. Chubukov, Phys. Rev. Lett. 83, 1652 (1999).

${ }^{15}$ M. Eschrig and M. R. Norman, Phys. Rev. B 67, 144503 (2003).

${ }^{16}$ K. Gofron, J. C. Campuzano, H. Ding, C. Gu, R. Liu, B. Dabrowski, B. W. Veal, W. Cramer, and G. Jennings, J. Phys. Chem. Solids 54, 1193 (1993).
${ }^{17}$ M. C. Schabel, C.-H. Park, A. Matsuura, Z.-X. Shen, D. A. Bonn, Ruixing Liang, and W. N. Hardy, Phys. Rev. B 57, 6090 (1998).

${ }^{18}$ M. C. Schabel, C.-H. Park, A. Matsuura, Z.-X. Shen, D. A. Bonn, Ruixing Liang, and W. N. Hardy, Phys. Rev. B 57, 6107 (1998).

${ }^{19}$ D. H. Lu, D. L. Feng, N. P. Armitage, K. M. Shen, A. Damascelli, C. Kim, F. Ronning, Z.-X. Shen, D. A. Bonn, R. Liang, W. N. Hardy, A. I. Rykov, and S. Tajima, Phys. Rev. Lett. 86, 4370 (2001).

${ }^{20}$ O. K. Andersen, A. I. Lichtenstein, O. Jepsen, and F. Paulsen, J. Phys. Chem. Solids 56, 1573 (1995).

${ }^{21}$ V. B. Zabolotnyy, S. V. Borisenko, A. A. Kordyuk, J. Geck, D. Inosov, A. Koitzsch, J. Fink, M. Knupfer, B. Buechner, S.-L. Drechsler, L. Patthey, V. Hinkov, and B. Keimer, arXiv:condmat/0608295v1 (unpublished).

${ }^{22}$ K. Nakayama, T. Sato, K. Terashima, H. Matsui, T. Takahashi, M. Kubota, K. Ono, T. Nishizaki, Y. Takahashi, and N. Kobayashi, arXiv:cond-mat/0611515v1, Phys. Rev. B (to be published).

${ }^{23}$ K. Nakayama, T. Sato, K. Terashima, H. Matsui, T. Takahashi, M. Kubota, K. Ono, T. Nishizaki, Y. Takahashi, and N. Kobayashi, Phys. Rev. B 75, 014513 (2007).

${ }^{24}$ H. L. Edwards, J. T. Markert, and A. L. de Lozanne, Phys. Rev. Lett. 69, 2967 (1992).

${ }^{25}$ S. H. Pan, E. W. Hudson, and J. C. Davis, Rev. Sci. Instrum. 70, 1459 (1999).

${ }^{26}$ M. Maki, T. Nishizaki, K. Shibata, K. Norio, and N. Kobayashi, J. Phys. Soc. Jpn. 70, 1877 (2001).

${ }^{27}$ B. W. Veal and Chung Gu, J. Electron Spectrosc. Relat. Phenom. 66, 321 (1994).

${ }^{28}$ N. Schroeder, R. Böttner, S. Ratz, E. Dietz, U. Gerhardt, and Th. Wolf, Phys. Rev. B 47, 5287 (1993).

${ }^{29}$ A. A. Kordyuk, S. V. Borisenko, A. N. Yaresko, S.-L. Drechsler, H. Rosner, T. K. Kim, A. Koitzsch, K. A. Nenkov, M. Knupfer, J. Fink, R. Follath, H. Berger, B. Keimer, S. Ono, and Yoichi Ando, Phys. Rev. B 70, 214525 (2004).

${ }^{30}$ J. M. Luttinger, Phys. Rev. 119, 1153 (1960).

${ }^{31}$ A. A. Kordyuk, S. V. Borisenko, A. Koitzsch, J. Fink, M. Knupfer, B. Büchner, H. Berger, G. Margaritondo, C. T. Lin, B. Keimer, S. Ono, and Yoichi Ando, Phys. Rev. Lett. 92, 257006 (2004).

${ }^{32}$ V. B. Zabolotnyy, S. V. Borisenko, A. A. Kordyuk, D. S. Inosov, A. Koitzsch, J. Geck, J. Fink, M. Knupfer, B. Büchner, S.-L. Drechsler, V. Hinkov, B. Keimer, and L. Patthey, Phys. Rev. B 76, 024502 (2007). 